\title{
DESIGN OF MOLECULAR SIEVE 4A USING COMPOSITE ASH: A STEP
}

\section{TOWARDS GREEN TECHNOLOGY}

\author{
S. Mane, S.U.Meshram* \\ *Department of Applied Chemistry, Laxminarayan Institute of Technology \\ R.T.M. Nagpur University, Nagpur \\ E-mail : siddhu_102@rediffmail.com
}

\begin{abstract}
The Chemical Composition of coal ash depends largely on quality of coal. The chief constituents of such ash are oxides of silicon, aluminum and iron with trace metals. Although the literature review reveals the usage of coal ash towards the high conversion of Zeolitic phases having wide applications in detergent, petrochemical and catalyst area; the concept of composite ash is quite fascinating. This includes the mixture of coal and rice husk in proper stoichiometry introduced in electric utility generating stations which produces nearly 30 to $40 \%$ ash with low emission of $\mathrm{CO}_{2}$. Molecular sieves or Zeolites on the contrary; are the hydrated nano materials having crossed linked network of silicon and aluminum ions bridged by oxygen linkages having uniform pore opening with high surface area. Since $85-90 \%$ composition of ash is oxides of silicon and aluminum hence it can be used as tailor made material for the production of Zeolites or molecular sieves.
\end{abstract}

The current paper deals with the attempts made in the direction for the extraction of amorphous silica from the ash in the form of sodium silicate and enriched with sodium aluminate prepared separately to produce a value added product Viz; Molecular sieve 4A commonly known as Zeolite-A. The crystalline Zeolitic phases in the samples were investigated using XRD, SEM and IR. A special emphasis has been given to optimize the reaction parameters in such a way that it would turns in to cost effective technology.

Keywords: Ash, Waste, $\mathrm{CO}_{2}$, Nano, Molecular Sieve

\section{INTRODUCTION:}

The amount of ash generated by coal-based thermal power plants has been increasing at an alarming rate throughout the world. The disposal of such a huge quantity of ash thus becomes a pressing issue. In India, more than 90 million tons of coal ash is generated annually being 
occupied by ash ponds with more than 65,000 acres of land ${ }^{\mathbf{1 - 2}}$. Several approaches have been made for proper utilization of ash, either to reduce the cost of disposal or to minimize the environmental impact. In our country, the majority of electrical energy is produced from coal hence the rate of consumption of coal from their reservoir is quite high. In this context, searching for a suitable alternative for coal in thermal power plants for energy generation is a need of hour. ${ }^{3}$ Although the number of power plants having more than 500 MW capacities are large in India; there exist many small power utility centers having capacity of 05 to 20 MW especially in Vidharbha region which is purely agro based. Recently rice husk as a potential source of renewable energy has received a significant attention. Since the calorific value of rice husk is almost half than that of coal; it is preferred to blend with coal to improve the energy production. During combustion it reduces the $\mathrm{CO}_{2}$ emission up to maximum extent since rice husk is a non carbonaceous source. The post combustion residue remains after the process is termed as composite ash, which factually represents the physico-chemical composition of coal and biomass simultaneously. It was also observed that the percent composition of this ash is neither matching with coal ash nor with pure biomass based ash. The production of composite ash is about $10-12 \%$ in these mini thermal power plants which offers an extra pollutant load upon air, water and land. ${ }^{4}$ Amongst the various utilization of rice husk ash, there is significant interest in its use in preparation of molecular sieve $4 \mathrm{~A}$ due to its widespread industrial uses in separation process as a sorbent, membrane, detergent builders and catalyst etc ${ }^{\mathbf{5}}$.

Most of the urban countries like Iran, Afghanistan, Thailand, Taiwan \& Japan; biomass based power generation units are already exists. Few reports are also available on process for production of molecular sieve 4A and other zeolitic phases from rice husk ash $\mathbf{6 - 8}$. No doubt, maximum researchers have identified coal ash as a potential source in the manufacturing process for molecular sieve 4A; however the concept of 
composite ash is quite new. ${ }^{\text {-12}}$ This includes mixture of rice husk $\&$ coal blended in to 60: 40 or 80: 20 ratio, where 60 or $80 \%$ represents rice husk. The stoichiometry is optimized according to availability of rice husk from farmers end $\&$ the present cost of coal.

Attempt has been made in this context to synthesize molecular sieve from waste composite ash and optimize the reaction parameters such as reflux time, temperature, crystallization period etc. Special emphasis has been focused in the era of recycling the alkaline mother liquor/ residueal ash to make the overall process green, cost effective \& ecofriendly.

\section{Materials and Method}

\section{Materials}

$\mathrm{NaOH}$ (purified flakes) and Aluminum hydroxide gel from Merck were used to extract sodium silicate and manufacture of Sodium aluminate. The CRHA (Composite Rice Husk Ash) was procured from different mini thermal power station of Vidharbha region of India.

\section{Methodology}

Sodium silicate was prepared by refluxing composite ash using 2.0 to 2.5 $\mathrm{N} \mathrm{NaOH}$ for 4 to $6 \mathrm{~h}$ at 100 to $120^{\circ} \mathrm{C}$. After filtration of clear sodium silicate solution, the residual ash was washed and recycled. Aqueous solution of Aluminum hydroxide was prepared separately by refluxing $\mathrm{NaOH}$ and aluminium hydroxide gel in deionized water. After this, with continuous stirring the measured quantity of aluminum hydroxide gel was blended in sodium silicate solution. The mixture was then allowed to aged for a period of $4 \mathrm{~h}$. The aged slurry was further subjected for hydrothermal crystallization in stainless steel autoclave of $500 \mathrm{ml}$ capacity for $3 \mathrm{~h}$ at $95-100^{\circ} \mathrm{C}$. The solid mass of molecular sieve was then filtered and washed with deionized water. The as such synthesized material was dried in oven at $55^{\circ}$ to $60^{\circ} \mathrm{C}$. 


\subsection{CHARACTERIZATION}

\subsection{X-ray Diffraction}

The powder X-ray diffraction (XRD) patterns of commercial molecular sieve 4A sample procured from Degussa, Germany and composite rice husk ash based molecular sieve sample was examined using an X-ray diffractometer and presented in Figure 1 and Figure 2 respectively. The major peaks of commercial standard were selected and compared with as such synthesized molecular sieve sample. The percent crystallinity was taken as the sum of the peak heights of the unknown materials divided by the sum of the peak heights of a standard material that has been assumed to be $100 \%$ crystalline i.e.

$\%$ Crystallinity $=$ (sum of the peak heights of unknown material) $/$ (sum of peak height of standard material) $\times 100$

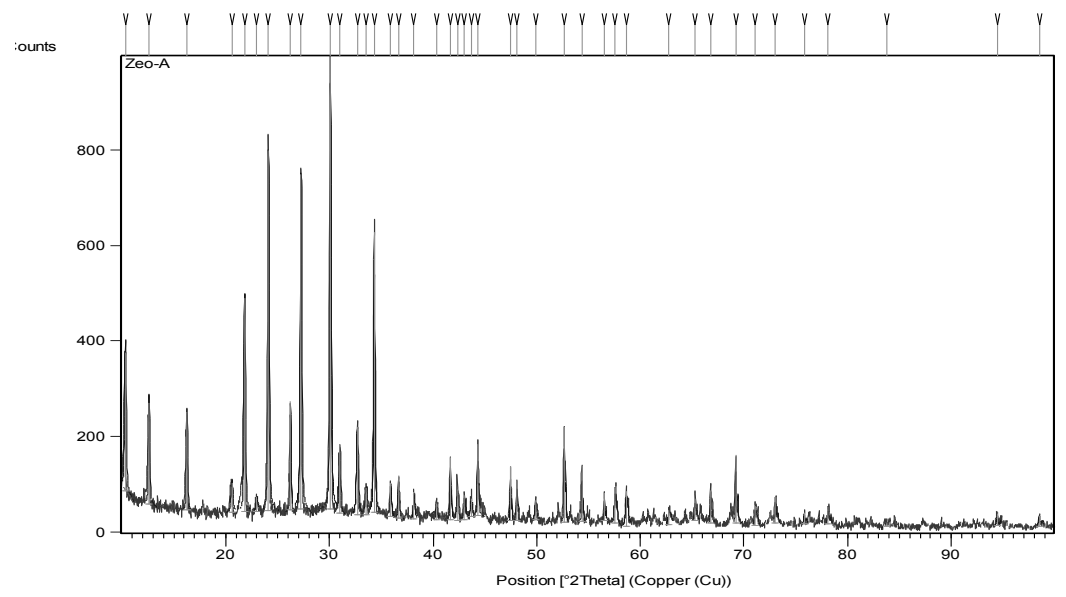

Figure 1: X- ray diffraction pattern of Commercial Molecular Sieve 4A. 


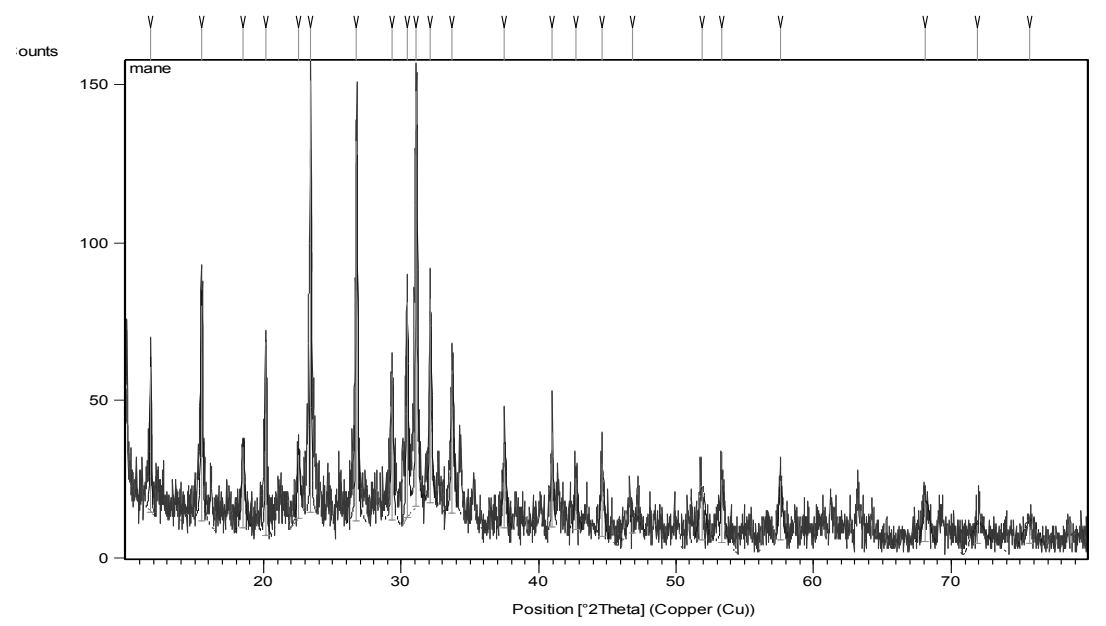

Figure 2: $\mathrm{X}$ ray diffraction pattern of CRHA based Molecular Sieve 4A.

\subsection{Scanning Electron Microscopy (SEM)}

The structural morphology of composite rice husk ash based molecular sieve 4A sample along with the commercial standard was studied using scanning electron microscopy and the photographic results are depicted in Figure 3 and Figure 4.

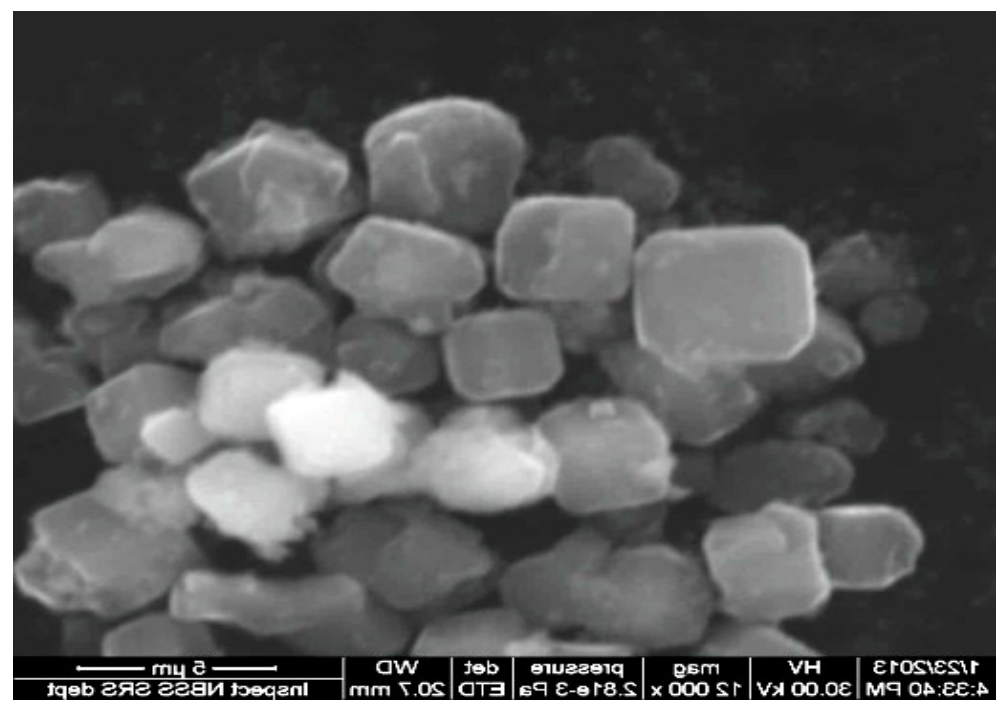

Figure 4: SEM of commercial Molecular sieve 4A. 


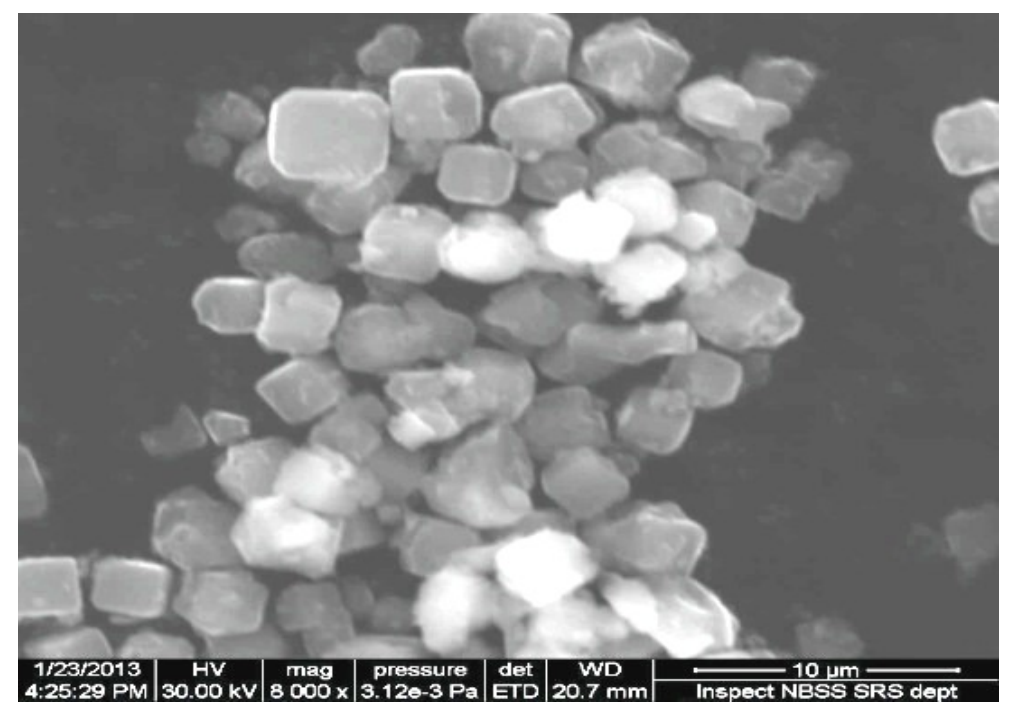

Figure 5: SEM of CRHA based Molecular sieve 4A.

\subsection{Fourier transforms infrared (FT-IR) spectra}

Infrared spectroscopic analysis for standard molecular sieve $4 \mathrm{~A}$ and CRHA based molecular sieve 4A sample has been conducted in the range of wave number $400-4000 \mathrm{~cm}^{-1} .^{13}$ The respective IR pattern is pointed in Figure 5 and Figure 6.

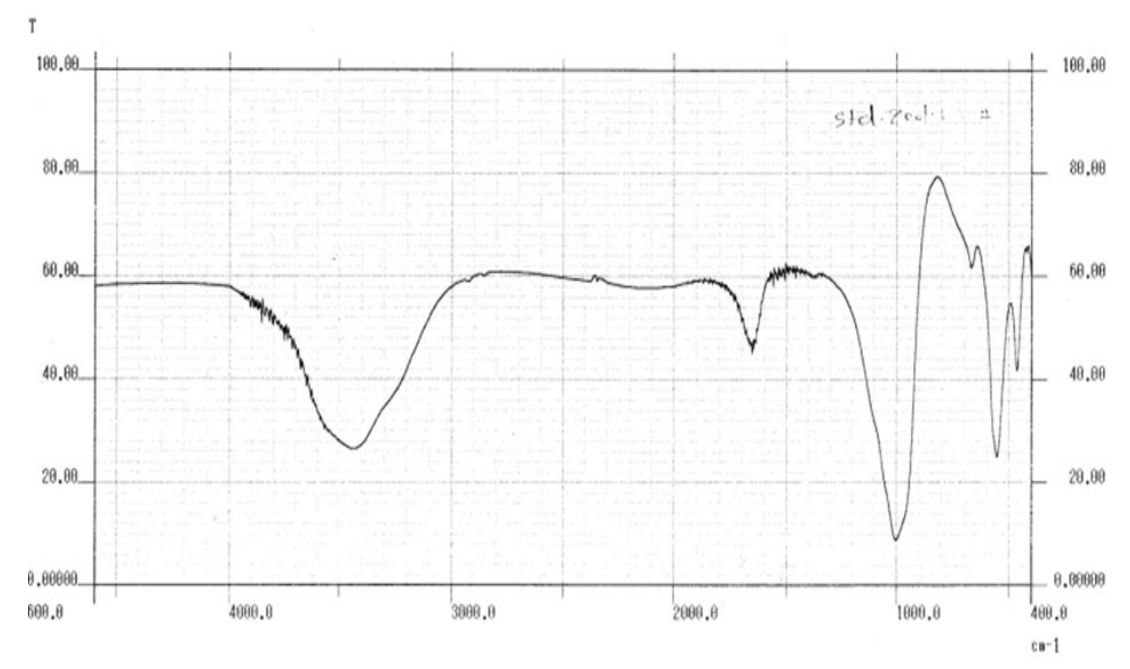

Figure 5: IR pattern of commercial Molecular sieve 4A. 


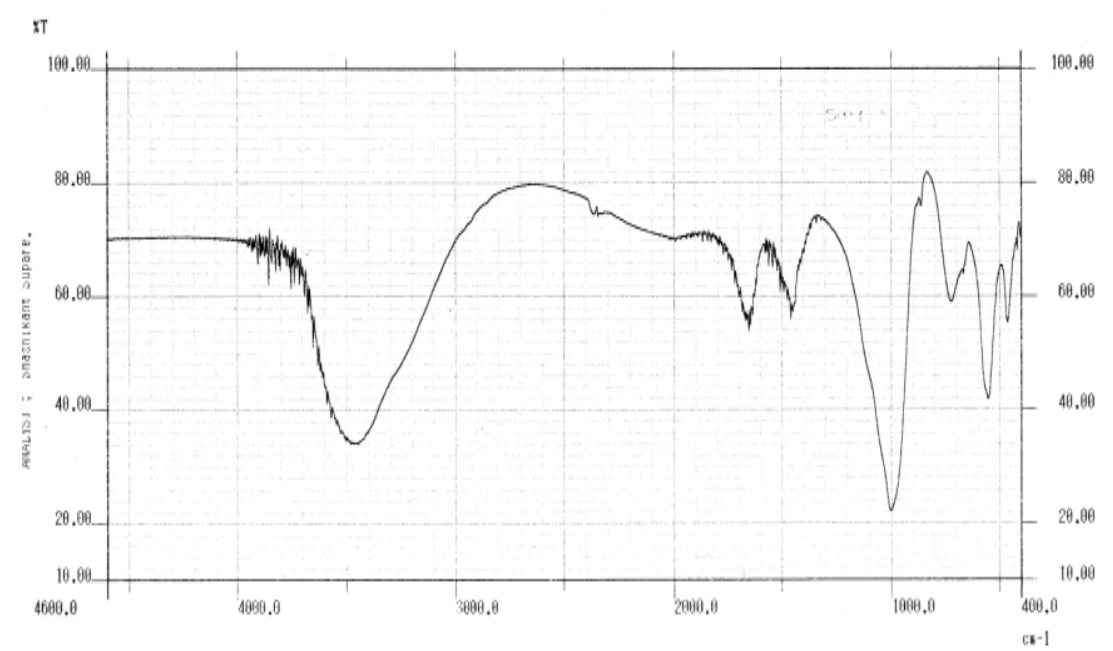

Figure 6: IR pattern of CRHA based Molecular sieve 4A

\section{RESULTS AND DISCUSSION}

\subsection{X-ray diffraction pattern}

The Sharpe peaks obtained in the diffractograms of both the samples matches significantly at glancing angle $2 \varnothing$. The percent crystallinity was calculated using the described methodology and it was noted that the CRHA based molecular sieve 4A resembles about $85 \%$ crystalline structure compared with commercial standard.

\subsection{Structural Morphology}

Figure 3 and Figure 4 clearly reveals that the structural morphology of commercial sample and CRHA based molecular sieve 4A are square planer and resembling closely with each other. The lack of amorphous phase in the Figure 4 pointing the highly crystalline nature Vis a vis purity of synthesized sample.

\subsection{Fourier Transform Infrared (FT-IR) spectra}

The comparative Infrared spectroscopic analysis for commercial standard with CRHA based molecular sieve has been recorded between the wave number of $400-4000 \mathrm{~cm}^{-1}$ respectively. Both the samples reveal important bands in the region of $1000-990 \mathrm{~cm}^{-1}$ represents O-Si-O, Si-O or Al-O stretching. The next strongest band at $480 \mathrm{~cm}^{-1}$ region is assigned to a 
Si-O or Al-O bending mode $\left(\mathrm{T}^{-} \mathrm{O}_{4}\right)$. A band in the $560 \mathrm{~cm}^{-1}$ region is related to the presence of double rings, D4R in the framework of nano molecular sieve 4A structure.

\section{CONCLUSION}

The results pertaining to the analytical studies towards the molecular sieve 4A sample are quite encouraging. It was also concluded that the composite rice husk ash would become a ideal source for tailoring the molecular sieve 4A on commercial basis. The steps included in the methodology are quite simple and non tedious so as to upscale the process commercially viable.

\section{ACKNOWLEDGEMENT}

The author acknowledge the support extended by Prof. T. B. Chahande, Director In-Charge, Laxminarayan Institute of Technology for his constant encouragement. The help and cooperation rendered by $\mathrm{Mr}$. Nikhil Dhote, Director NCSR chemical's private 1td. for collecting the ash samples from various mini power station in the Vidharbha region is highly appreciated. Thanks are due to the staff of National Bureau of Soil Survey (NBSS), Nagpur, Post graduate teaching Department of Physics and Pharmacy for SEM, XRD and IR characterization of Molecular sieve 4A samples.

\section{REFERENCES}

R. Bagchi \& A. Ghosh "International conference on fly ash disposal \& utilization",20-22 January , New Delhi, 1, 1998; 45

R, Maunder \& S. Sarkar; "International conference on fly ash disposal \& utilization", 20-22 January, New Delhi, 1, 1998; 45.

Gidde M., and Jivani A., (2007), Waste to wealth-potential of rice husk in India a literature review". Procceding of the International conference on cleaner technology and environment management. 4-6 January, Pondicherry, India ,586-590 
Islam M. N., and Ani F. N., (2000).Techno-economics of rice husk pyrolysis, conversion with Catalytic treatment to produce liquid fuel", Bioresource technology; 67-75.

Petkowicz. D. I., Ring R. T., Redtke C., Pergher S. B. and Dos Santos, J.H. Z. (2008). "Zeolite Na from Brazilian chrysotile \& Rice Husk". Microporous \& Mesoporous Mater. 116(1-3): 548 -554.

Hani, N. (2001) "Direct Synthesis of $\mathrm{Na}$ Zeolite from rice husk \& carbonaceous rice husk ash". Indonesian J. Agric. Sci. (I) : 40-45

Soto, K., Sugimoto, K. and Nakane, T. (2008). Synthesis of industrial scale NaY zeolite membranes and ethanol performance in vapor permeation up to $130^{\circ} \mathrm{C}$ and $570 \mathrm{kPa}$. J. Membr. Sci.310 (1-2): 161173.

Chayakorn B. \& pesak R, (2009). "Synthesis of Zeolite-A Membrane from Rice Husk Ash", Journal of Metals, Material \& Minerals. Vol. 19; 79- 83.

Rayalu, S., Labhsetwar N. \& P. Khana; (1998). "Production of zeolite -A from fly ash" US patent No.5965105.

Rayalu, S., Labhsetwar N \& P. Khana; (2000). "Production of zeolite $-Y$ from fly ash". US patent No.6027708.

Rayalu, S.S., Udhoji J.S., Munshi, KN., and Hasan, M.Z. (2001). "Highly crystalline zeolite- A from fly ash of bituminous and lignite coal combustion. Journal of Hazardous Materials, 107-121.

Hui K.S.Hui., and Chao C.Y.H. (2006). Effect of step-change of synthesis temperature on synthesis of zeolite 4A from coal fly ash. Microporous and Mesoporous material, 145-151.

Barrer, R.M., (1982) "Hydrothermal Chemistry of Zeolite". Academic press, London. 